\title{
LA DISTINCIÓN DOXÁSTICO-SUBDOXÁSTICO
}

\author{
LIZA SKIDELSKY \\ Departamento de Filosofía \\ Universidad de Buenos Aires \\ Consejo Nacional de Investigaciones Científicas y Técnicas \\ lskidelsky@filo.uba.ar
}

\begin{abstract}
RESUMEN: En este trabajo discuto los criterios que se han postulado para establecer una distinción entre los estados doxásticos y los subdoxásticos; a saber: accesibilidad a la conciencia (Stich 1978), integración inferencial (Stich 1978) y conceptualización (Davies 1986, 1989). Esta discusión se realiza a través del análisis de los argumentos propuestos por Davies (1989). Mi tesis es que si bien habría una distinción intuitiva entre ciertas clases de estados mentales, estos criterios, según las dos lecturas posibles de lo que el mismo Davies propone para que un criterio constituya una distinción de principio, no parecen establecer la distinción deseada.
\end{abstract}

PALABRAS CLAVE: estados del procesamiento de la información, estados intencionales, contenido no conceptual, distinción personal-subpersonal

SUMMARY: In this paper I discuss the criteria that have been postulated for a principled distinction between doxastic states and subdoxastic states, namely: accesibility to consciousness (Stich 1978), inferential integration (Stich 1978), and conceptualization (Davies 1986, 1989). The discussion set off from the analysis of the arguments put forward by Davies (1989). My claim is that although there would be an intuitive distinction among certain kinds of mental states, according to two plausible readings of what Davies himself proposes as a principled criterion, such criteria do not seem to establish it.

KEY WORDS: information-processing states, intentional states, non-conceptual content, personal-subpersonal distinction

A fines de los años 1970, Stich propuso una fundamentación de una "distinción intuitiva" entre dos clases de estados psicológicos, con el objetivo de mostrar que esta distinción marca un límite psicológico real e interesante, y que esto trae consecuencias para la teorización en ciencia cognitiva. Stich distinguió entre estados intencionales, como los de creencia, y estados que "desempeñan un papel en la historia causal próxima de las creencias, aunque en sí mismos no son creencias" (1978, p. 499), y a estos últimos los llamó estados subdoxásticos (ES). Los ES son estados psicológicos que almacenan información acerca de, por ejemplo, las reglas de la gramática que llevan a juicios de gramaticalidad o los rasgos de la imagen retinal que dan lugar a juicios perceptuales. En ambos casos hay mecanismos de procesamiento de la información que median entre el estímulo (auditivo o visual, por ejemplo) y la creencia, cuya tarea consiste en utilizar la 
información almacenada en sus estados para dar lugar (seguramente junto con otros mecanismos cognitivos) a la creencia correspondiente.

Estos estados forman parte del proceso de formación de creencias sin ser ellos mismos creencias. De manera que habría una diferencia entre la creencia de que $p$ y el estado que almacena o representa la información que $p$, aunque ambos puedan tener el mismo contenido p. Stich ofreció dos criterios para distinguir los ES de los estados intencionales (EI): la accesibilidad a la conciencia y la integración inferencial, y trató de mostrar que los ES son inaccesibles a la conciencia y están aislados inferencialmente. Si este análisis era correcto, esto significaba que la ciencia cognitiva debía tomar en cuenta esta distinción como un camino para, por un lado, evitar líneas de investigación que llevaran al fracaso — por ejemplo, seguir desarrollando modelos inferenciales para los ES - y, por otro, abordar cuestiones fructíferas como preguntarse por qué la integración inferencial y la accesibilidad a la conciencia van juntas, si hay un mecanismo común subyacente a ambos rasgos, etcétera.

Davies (1986, 1989) retoma los criterios propuestos por Stich y agrega un tercero a partir de la noción de "contenido no conceptual" propuesta por Evans (1982). Davies defiende la idea de que los dos rasgos anteriores no se sostienen por sí mismos y que están fundamentados en el criterio de conceptualización; por ende, es realmente este último el que carga el peso de la distinción entre los ES y los EI. Al igual que Stich, Davies cree que ambos tipos de estados tienen contenido semántico y participan en explicaciones causales, y que hay una distinción teórica y psicológica importante entre los estados de procesamiento de la información y las actitudes proposicionales. En particular, esta diferencia permite, según Davies, dilucidar la naturaleza de los estados de conocimiento semántico que son tácitos.

La perspectiva general desde la cual los filósofos ofrecen los criterios de (a) accesibilidad a la conciencia (Stich 1978), (b) integración inferencial (Stich 1978), y (c) conceptualización (Davies 1986, 1989) no es a partir de consideraciones empíricas sino a partir del análisis del concepto ordinario preteórico de "creencia". l Es este análisis

${ }^{1}$ Sería más correcto formular los criterios de esta manera: accesibilidad/inaccesibilidad a la conciencia, integración/no integración inferencial y contenido conceptual/contenido no conceptual; (a), (b) y (c) serán, respectivamente, la manera sucinta de decirlo. Por otro lado, cabe hacer notar que el rasgo (a) es producto de motivaciones tanto a priori como empíricas. Propuesto inicialmente por Chomsky, (a) está motivado por consideraciones empíricas, en particular, y tal como veremos más adelante, por argumentos a la mejor explicación. En las versiones de Stich 1978 y Davies 1989, la motivación es puramente a priori. 
a priori el que pretende justificar la "distinción intuitiva" entre los EI y los ES. El análisis del concepto ordinario de "creencia" muestra que el individuo que posee creencias tiene conciencia del contenido de sus creencias, que éstas están inferencialmente integradas, y que sus contenidos son conceptuales. Mientras que, con respecto a los ES, el individuo no tiene acceso consciente a sus contenidos, están aislados inferencialmente y sus contenidos son no conceptuales.

En lo que sigue analizaré estos criterios, a través de la discusión de los argumentos de Davies 1989. Mi tesis es que si bien habría una distinción intuitiva entre ciertas clases de estados mentales, estos criterios no parecen establecer la distinción deseada. A grandes rasgos, mientras que estoy de acuerdo con Davies en que (a) y (b) no permiten, por sí mismos, establecer la distinción (cuestión de la que no me ocuparé en este trabajo), no estoy de acuerdo en las razones particulares que ofrece en contra de estos criterios (secciones 1-2), ni en la razón fundamental que afirma que (a) y (b) no se mantienen por sí mismos sino que se fundamentan en (c) y, en este sentido, (c) permitiría establecer una distinción de principio (sección 3). Contra esta razón fundamental intentaré sostener que, al menos en las dos lecturas posibles de lo que el mismo Davies propone para que un criterio constituya una distinción de principio, (c) no satisface ninguna. De manera que para rescatar una distinción interesante, para la ciencia cognitiva, entre clases de estados mentales, habría que recurrir, quizá, a otra distinción (sección 4).

\section{Accesibilidad a la conciencia}

A partir de los primeros desarrollos de la teoría chomskiana, la inaccesibilidad a la conciencia está ligada a los mecanismos y estados cognitivos de la lengua- $\mathrm{I}^{2} \mathrm{y}$, desde entonces, Chomsky se ha encargado de defender, en (casi) todos sus escritos, la idea de que aquéllos no sólo no son efectivamente accesibles a la conciencia, sino que ni siquiera son potencialmente accesibles a la conciencia (por ejemplo, Chomsky 1965, p. 8; 1980a, pp. 137-142; 1986, p. 260, pp. 269-271; 1994, pp. 93 y ss.). El sentido de conciencia al que Chomsky alude es en términos de poder informar algo, asentir a ello, o reflexionar al

${ }^{2}$ La lengua-I junto con los sistemas de ejecución conforman la facultad del lenguaje. La lengua-I consiste en un mecanismo computacional y un léxico: el procedimiento computacional genera descripciones estructurales de las expresiones lingüísticas a partir de elecciones léxicas. El output de la lengua-I actúa como instrucciones para los sistemas de ejecución: el articulatorio-perceptual y el conceptual-intencional, que lo interpretan y siguen sus instrucciones para el pensamiento y la acción (Chomsky 1995a, 1995b). 
respecto. Los hablantes no pueden, en general, reportar sus estados lingüísticos y, cuando lo intentan, la mayoría de las veces suelen estar equivocados, de manera que los contenidos no están abiertos a la reflexión. Además, los hablantes no reconocen ni asienten cuando son informados acerca de las representaciones mentales involucradas en el uso del lenguaje; de modo que no hay acceso por introspección a los mismos.

Esto sería evidencia a favor de que los estados lingüísticos son inconscientes. Pero, en realidad, el argumento fundamental en defensa de que hay estados y mecanismos inconscientes tiene la forma de los argumentos a la mejor explicación. Dado cierto fenómeno que se pretende explicar y dado que, de todas las explicaciones en competencia, la explicación que postula estados y procesos inconscientes es la mejor, según ciertos criterios de elección entre explicaciones, es probable que esta explicación sea la adecuada. El criterio de elección al que se suele aludir es el poder explicativo de la teoría, y no parece haber ninguna explicación que, sin postular un mecanismo inconsciente, pueda dar cuenta de los numerosos hechos que explica una teoría que los postula (Chomsky 1980a, pp. 139-140). ${ }^{3}$ Otro criterio al que se suele apelar es el de la familiaridad o concordancia con tipos de explicaciones aceptadas; así, la explicación que postula estados y procesos inconscientes concuerda con el tipo de explicación habitual de las ciencias cognitivas que postula que gran parte del procesamiento de la información ocurre en un nivel inconsciente.

Desde el punto de vista del análisis del concepto ordinario de "creencia", la conclusión es la misma: los ES son inconscientes. Stich (1978), al igual que Chomsky, analiza la accesibilidad a la conciencia de las creencias y sus contenidos en términos de su disponibilidad para el informe verbal explícito. Un individuo puede decir tanto si cree que $p$ (es decir, informar de su creencia) como, si cree que $p$, informar que $p$ es el caso (es decir, expresar y asentir a su contenido). Y esto es así en virtud de estar en ese estado. En

\footnotetext{
${ }^{3}$ Tales como la dependencia referencial. Por ejemplo, dada dos oraciones (1) $\mathrm{He}$ thinks Bill is a nice guy (Él piensa que Bill es un muchacho agradable), y (2) The woman he married thinks Bill is a nice guy (La mujer que se casó con él piensa que Bill es un muchacho agradable), un hablante conoce de manera inconsciente que en (1) él no depende referencialmente de Bill (no se interpreta (1) como "Bill piensa que Bill es un muchacho agradable"), mientras que en (2) es posible la dependencia referencial (se puede interpretar (2) como "La mujer que se casó con Bill piensa que Bill es un muchacho agradable"). Esto se explica porque el estado inicial de la facultad del lenguaje tiene principios relacionados con la dependencia referencial (que son estudiados por la Teoría del Ligamiento, Chomsky 1994, p. 93).
} 
cambio, los ES y sus contenidos no son accesibles a la conciencia en el sentido de que el individuo no puede informar de ellos ni puede asentir a los mismos. Como se puede notar, tanto Chomsky como Stich consideran el fenómeno de la conciencia en términos de poder reportar y asentir. ${ }^{4}$

Según Davies (1989), esta noción preteórica de "accesibilidad a la conciencia" no es adecuada como criterio de principio para la delimitación entre los ES y los EI por tres razones: 1) hay creencias inconscientes; 2) hay aspectos de la red inferencial de creencias que son inconscientes, y 3) el ámbito de la experiencia consciente es más amplio que el de los EI. Parece ser que las dos primeras razones permitirían sostener que ser inconsciente no es condición suficiente para ser un ES, mientras que la tercera establecería que ser inconsciente no es una condición necesaria para ser un ES. Si bien estoy de acuerdo con Davies en que el criterio (a) de accesibilidad a la conciencia no marca una delimitación de principio (cuestión que no argumentaré aquí), sus razones no me parecen, en general, buenas razones. Veámoslas en orden.

El argumento que subyace a 1 podría reconstruirse de la siguiente manera: tenemos una noción de "creencia inconsciente", si no se quiere que colisione con la noción de "ES", habría que poner restricciones. Se podría restringir la noción de "creencia inconsciente" utilizando una variante del principio de que cualquier EI es inconsciente sólo en el sentido de que, en principio, es accesible a la conciencia salvo un mecanismo de bloqueo. Pero esto no sería suficiente porque se corre el riesgo de clasificar casos paradigmáticos de ES como estados no accesibles a la conciencia por un mecanismo de bloqueo, con lo cual, la noción de "ES" colisionaría, otra vez, con la de "creencia inconsciente". Por ende, habría que restringir aún más este principio, pero parece que cualquier restricción que se piense, probablemente, constituiría un criterio independiente, de (a), para fundamentar la distinción.

\footnotetext{
${ }^{4}$ No obstante, véase la distinción entre conocimiento inconsciente y conocimiento consciente no verbalizable en Chomsky 1986, p. 271. Por otro lado, Stich también dice que, en los casos típicos de creencia, el sujeto es consciente de los contenidos en el sentido de tener la experiencia consciente de "tener la creencia ocurrente de que $p "$ (p. 504). Me parece que atribuir a los EI propiedades de la conciencia fenoménica, además de propiedades de la conciencia de acceso (véase la distinción en Block 1995), conduciría, en este punto, a cierta confusión. Dado que el tipo de conciencia que está en juego en esta discusión acerca de la naturaleza de los estados cognitivos es la de acceso y dado que es discutible que estados como los de creencia tengan propiedades fenoménicas, es conveniente tomar la noción de "conciencia" en el sentido de acceso ( $c f r$. Davies 1995 para ambos puntos).
} 
Mi primera observación consiste en hacer notar que el argumento de Davies descansa en la aceptación de que puede haber algo así como creencias inconscientes. Un defensor de (a) podría negar esta posibilidad, y así (a) sería un criterio delimitador de principio. No obstante, esta estrategia no funcionaría porque parece difícil negar que, al menos, tenemos una noción de sentido común de "creencia inconsciente". El defensor del criterio podría responder a esto que lo que está en juego no son nociones de sentido común (difícilmente se tenga una noción de sentido común de "ES"), sino nociones teóricas, en el sentido de que participan en teorías, en particular, las psicológicas. Si esto no fuera así, seguiría el argumento del defensor, no se ve por qué la distinción doxástico-subdoxástico tendría que tener importancia para las teorías psicológicas. Así, mientras que la noción de "mecanismos y estados de procesamiento de información inconscientes" participa, al menos, de teorías bien establecidas como las del lenguaje (Chomsky) y la visión (Marr), en cambio, es dudoso que la noción de "creencia inconsciente" participe de una teoría bien establecida. Aunque haya quienes consideran (con reparos) que esta noción es clave en la teoría psicoanalítica (Stich 1978, Searle 1990 y Davies 1989), no está claro que en ella se hable de creencias inconscientes. La noción controvertida, concluye el defensor, no es el adjetivo "inconsciente", sino la asociación del mismo a las creencias, dado que no está claro que el psicoanálisis utilice la misma noción de "creencia" que la desarrollada por la filosofía de la mente (aunque aparezca el término "creencia" en la teoría psicoanalítica). No es éste el lugar para un análisis de la teoría freudiana; mi intención es sólo señalar que la aceptación de la noción teórica (no la de sentido común) de "creencia inconsciente" requiere una elaboración que Davies no le dedica en su crítica de (a).

Mi segunda observación apoyaría la conclusión de Davies si lo que ésta quiere decir es que cualquier restricción a la noción de "creencia inconsciente" estaría anclada en un criterio que no es el de accesibilidad a la conciencia. Una manera que se me ocurre de entender esto sería la siguiente. Si, aún a pesar de lo que he dicho antes, resultara que hay una noción de "creencia inconsciente" que desempeña un papel en las teorías cognitivas y si lo único que distingue las creencias de los ES es la accesibilidad a la conciencia, sería plausible suponer que, por ejemplo, se considera que la naturaleza de la creencia, consciente o inconsciente, y la de los ES es la misma. De modo que, si tener una creencia consiste, por ejemplo, en estar en una relación computacional con representaciones almacenadas, tener 
un ES también consistiría en tener algún tipo de relación computacional con representaciones almacenadas. En este sentido, podría ser que las creencias inconscientes se distinguieran de los ES (y de las creencias conscientes) por el tipo de actitud que se mantiene con el contenido; quizá una actitud distinta de las hasta ahora conocidas (por ejemplo, creencia*). Pero está claro que esta restricción ya no estaría anclada en la accesibilidad a la conciencia, sino que se basaría en un criterio independiente, relacionado con la naturaleza de las creencias inconscientes. En ese sentido, creo que cualquier intento de restringir la noción de "creencia inconsciente" llevaría a un criterio distinto de (a).

La estrategia que utiliza Davies no es, entonces, restringir la noción de "creencia inconsciente", sino refinar la noción de "accesibilidad a la conciencia". Esta noción habría que entenderla no como disponibilidad para el informe verbal, sino como disponibilidad de los contenidos para el pensamiento. Así, un contenido es accesible a la conciencia si en virtud de estar en ese estado, el sujeto es capaz de tener pensamientos con ese contenido y, para ello, se requiere que el contenido esté conceptualizado. Esta estrategia también lleva a basar (a) en otro criterio: el de conceptualización (c), pero éste no sería independiente de (a) porque lo fundamentaría; en última instancia, (a) se basa en (c) y, en este sentido, es (c) el que establece la distinción de principio. Dado que veremos, en la sección 3, que (c) no establece una distinción de principio, la estrategia de Davies en contra de que (a) pueda establecer esa distinción se queda sin sustento.

La razón 2, esto es, que hay aspectos de la red inferencial de creencias que son inconscientes, descansa en la observación de Fodor (1983, p. 85) de que un individuo puede realizar inferencias que son instancias del modus ponens, puede ofrecer las premisas como razones para la conclusión y, sin embargo, no puede formular la regla en su generalidad. Según Davies, nuestro compromiso con la regla de inferencia, aunque ésta sea inconsciente, no parece un caso prima facie del dominio subdoxástico. Davies ensaya dos respuestas imaginarias de los defensores de (a), basadas en la distinción entre estados representacionales y procesos computacionales. La primera sostiene que la accesibilidad a la conciencia es un rasgo de los estados y no de los procesos, de manera que el hecho de que haya reglas inconscientes en la red inferencial de creencias no amenaza el criterio de accesibilidad a la conciencia. Davies le objeta a esta defensa que, de esta manera, el rasgo de inaccesibilidad a la conciencia de, por ejemplo, las reglas lingüísticas, que según él, muy probablemente se instancian como procesos computacionales más que como estados representacionales, 
no sería de peso para delimitar el ámbito subdoxástico porque habría reglas que intervienen en el ámbito intencional que también serían inconscientes y, de este modo, (a) no cumpliría ningún papel delimitador y, con ello, se desdibujaría la distinción entre el ámbito intencional y el subdoxástico.

Lo que creo que está diciendo Davies es que si se le quita peso delimitador al criterio de accesibilidad a la conciencia aplicado a las reglas (operaciones, procesos) y, con ello, se restringe (a) sólo a los estados, entonces el principal argumento, del defensor de (a), por el cual considerar como subdoxástico al ámbito del procesamiento lingüístico temprano, se derrumba. Porque, según parece sostener Davies, el carácter no accesible a la conciencia de las reglas lingüísticas sería lo que supuestamente volvería subdoxástico el ámbito del procesamiento lingüístico temprano. En lo que sigue intentaré ofrecer razones para pensar que mientras haya estados lingüísticos en el ámbito subdoxástico, el criterio de inaccesibilidad a la conciencia, aplicado a los estados, seguiría sirviendo como criterio delimitador del ámbito lingüístico subdoxástico.

En primer lugar, si se creyera que lo único subdoxástico en la teoría chomskiana son las reglas, entonces sería cierto que el criterio de accesibilidad a la conciencia no distinguiría el ámbito subdoxástico del intencional porque habría reglas inconscientes en los dos ámbitos. Pero no puede ser el caso porque, aunque las reglas estén instanciadas en los mecanismos de procesamiento computacional, éstos requieren operar sobre algo; esto es, no habría computaciones sin objetos sobre los cuales computar, de manera que los procesos requieren operar sobre información, entendida, por ejemplo, como instancias de representaciones. Estas representaciones tienen que ser explícitas, porque los procesos están definidos sobre la información lingüística que portan estas representaciones. Los procesos pueden no estar explícitamente representados pero, los estados, en cuanto episodios en un proceso, tienen que estarlo (al menos, los outputs del mecanismo computacional lingüístico, i.e., los pares $\{\mathrm{FON}, \mathrm{SEM}\}$ de cada expresión lingüística de una lengua, son estados representacionales explícitos). ${ }^{5}$ Estas representaciones son las que figuran en los estados cognitivos subdoxásticos, de manera que la información que extraen los algoritmos computacionales para poder realizar sus

${ }^{5}$ FON(E) es una representación de la Forma Fonética que contiene información relevante para el sonido de una expresión lingüística $\mathrm{E}$, y $\mathrm{SEM}(\mathrm{E})$ es una representación de la Forma Lógica que porta información relevante al significado de E. FON(E) y $\operatorname{SEM}(\mathrm{E})$ son interpretadas por los sistemas articulatorio-perceptual y conceptualintencional, respectivamente.

Crítica, vol. 39, no. 115 (abril 2007) 
operaciones sobre las representaciones es la información que aparece en los ES. Así, la distinción doxástico-subdoxástico para los estados se sigue manteniendo más allá de que las operaciones sobre los ES o los EI no sean accesibles a la conciencia.

En segundo lugar, en el programa minimalista (Chomsky 1995a, pp. 5-6) no hay reglas para formar construcciones gramaticales (como en la gramática generativa de etapas anteriores); más bien, la información lingüística se instancia en principios y parámetros. Chomsky acepta la hipótesis de que los principios parametrizados de la gramática universal no se relacionan con el mecanismo computacional, sino con el léxico; cada parámetro refiere a propiedades de elementos específicos del léxico, en particular, morfológicos (Chomsky 1995a, p. 31). Así, mientras que las operaciones del sistema computacional se reducen básicamente a la operación de combinar (merge), operación que en sí misma no tendría nada esencialmente lingüístico, los principios parametrizados forman parte de la información con la que opera el sistema, de modo que lo estrictamente lingüístico estaría más en la información (léxica) que en los procesos (Chomsky 2005) y, en este sentido, es adecuado considerar que son los estados del sistema los que son pertinentes para la aplicación del criterio.

En conclusión, aun suponiendo que la información lingüística se instanciara en procesos, y no en estados, las reglas tienen que operar sobre algo y eso sobre lo que operan, i.e., las representaciones mentales, son los constituyentes de los estados, de manera que la aplicación de (a) a los estados seguiría siendo un criterio delimitador. Además, si se toman en cuenta modelos lingüísticos recientes, lo estrictamente lingüístico - esto es, aquello en lo que residiría la información lingüística - serían los estados y no los procesos que operan sobre esos estados, de manera que la aplicación de (a) a los estados seguiría siendo un criterio delimitador.

La segunda respuesta imaginaria de los defensores de (a), propuesta por Davies, sostiene que si el rasgo en cuestión provee un criterio claro de distinción entre los EI y los ES, entonces también contribuye a una distinción entre, por un lado, reglas mentales en el dominio intencional y, por el otro, procesos computacionales en el dominio subdoxástico. Tal como se desprende de lo dicho más arriba, con respecto a la primera respuesta, creo que el locus de la distinción son los estados. De todas formas, Davies contesta acertadamente que la posibilidad de extender de manera derivada la distinción a los procesos depende de si el criterio provee una distinción entre estados y, para mostrar que esto no es así, su razón 3 apunta directamente a 
los estados. Dado que, como se verá a continuación, el argumento de Davies que avala 3 no parece adecuado, sus objeciones basadas en la razón 2 (i.e., hay aspectos de la red inferencial de creencias que son inconscientes) se quedan sin el apoyo necesario para rechazar (a).

La razón 3, esto es, que el ámbito de la experiencia consciente es más amplio que el de los EI, se basa en un caso imaginado: supongamos que cierto aspecto de la experiencia consciente depende, de una manera sistemática, del contenido de cierto ES, que podría emerger a la conciencia (por ejemplo, como un pinchazo). Sin embargo, concluye Davies, esto no convertiría ese aspecto de la experiencia en candidato a creencia. Ahora bien, concediendo que algo como esto tuviera alguna plausibilidad empírica, en primer lugar, si lo que Davies quiere decir es que si los ES fueran conscientes, entonces tendrían que convertirse en creencias (puesto que lo único que distinguiría a los ES de las creencias es que los primeros son inconscientes), me parece que el caso imaginado no es el adecuado. Lo que pone en juego este caso imaginado no es lo que interesa, esto es, la conexión entre ES y las creencias, sino la relación entre las experiencias y las creencias. Un ES de procesamiento de la información puede dar lugar a una experiencia (perceptual o propioceptiva) que, a su vez, puede o no dar lugar a una creencia (perceptual o propioceptiva). Este caso imaginado es acerca de experiencias, y se sabe que las experiencias pueden no llevar a ninguna creencia o llevar a incredulidad (no dar crédito a lo que se percibe, por ejemplo, en las ilusiones ópticas, McDowell 1994, cap. 3, Martin 1994). Si imaginamos, tal como pide Davies, que un aspecto de esa experiencia esté asociado a un ES, ese aspecto subdoxástico de la experiencia, por más que sea consciente, no sería sin más candidato a creencia, simplemente porque las experiencias son independientes de las creencias, en el sentido mencionado.

En segundo lugar, si lo que está en juego es simplemente el acceso a la conciencia de un aspecto subdoxástico de la experiencia (sin que intervenga la noción de "creencia"), un defensor del criterio podría sostener, como afirma Davies, que a pesar de que el estado informacional puede ser accesible a la conciencia, su contenido no lo es. Davies (1995) sostiene que decir esto ya es refinar la noción preteórica de "accesibilidad a la conciencia" y que cualquier intento interesante finalmente apelará al criterio de conceptualización. He mencionado, respecto de la razón 1, que la accesibilidad interesante, según Davies, consistía en la disponibilidad de los contenidos para el pensamiento y dado que pensar requiere poseer conceptos, entonces la accesibilidad de los contenidos a la conciencia se fundamenta en que éstos 
estén conceptualizados. Cabe recordar que la estrategia general de Davies consiste en fundamentar los criterios (a) de accesibilidad a la conciencia y (b) de integración inferencial en el criterio (c) de conceptualización, de manera de sostener que (c) es el que permite una distinción de principio. También he mencionado que la viabilidad de esta respuesta depende de que (c) establezca realmente la distinción buscada y veremos, en la sección 3, que éste no es el caso.

\section{Integración inferencial}

Las creencias están relacionadas entre sí de manera que conforman una red de inferencias potenciales; los caminos por los cuales una creencia puede dar lugar a otra son innumerables a tal punto que casi cualquier creencia puede llevar a cualquier otra ("promiscuidad inferencial", Stich 1978), están al servicio de distintos propósitos (Evans 1981a) y pueden ser usadas (combinadas con deseos) como razones para la acción (Evans 1982, cap. 5; Davies 1989). En cambio, los ES no pueden combinarse con deseos para ser usados como razones para la acción, y están aislados inferencialmente del sistema de creencias de un individuo, en el sentido de que tienen un espectro limitado y empobrecido de patrones inferenciales posibles para dar lugar a creencias o ser producto de ellas y, en este sentido, no estarían al servicio de distintos propósitos.

No es que los ES no participen de relaciones inferenciales, sino que las relaciones están empobrecidas, de manera que los ES están parcialmente aislados de la red inferencial de creencias. Por un lado, los ES no se combinan con creencias para dar lugar a otras creencias. Un lingüista que tiene una creencia explícita acerca de una regla (o principio) puede realizar inferencias en conjunción con otras creencias, pero un hablante que sólo tiene información gramatical meramente almacenada no puede utilizar esta información, junto con otras creencias, para dar lugar a otra creencia. Así, por ejemplo, la creencia de que si se da la regla $r$, entonces Chomsky está seriamente equivocado, junto con el ES que almacena $r$, no lleva a la creencia de que Chomsky está seriamente equivocado (el ejemplo es de Stich 1978). En términos más generales, la creencia de que si $P$ entonces $Q$ combinada con el ES que $P$, no da lugar a la creencia de que $Q$, como sí podría hacerlo si ambas premisas fueran creencias. Por otro lado, los ES no se combinan con creencias para dar lugar a otros $\mathrm{ES}$, porque los sistemas que operan con ES no son cognitivamente penetrables por las creencias y, en general, el conocimiento del individuo. Las relaciones inferenciales entre los ES y las creencias son 
especializadas y restringidas porque los ES son parte de subsistemas cognitivos de propósito específico y, en este sentido, las relaciones inferenciales entre los ES de distintos subsistemas también son limitadas (y quizás inexistentes, en algunos casos).

La estrategia de Davies no es argumentar en contra del criterio de integración inferencial. Hacer esto podría haber consistido en argumentar en contra de que los ES no estén integrados inferencialmente con la red de creencias, o bien mostrar que la red de creencias no está integrada inferencialmente de la manera optimista en la que se cree que está integrada (promiscuidad inferencial), o hacer ambas cosas. Davies no está interesado en atacar el criterio (b) de integración inferencial sino que su estrategia consiste, más bien, en aceptarlo y sugerir que el hecho de que los contenidos estén conceptualizados es lo que permite dar cuenta de la integración inferencial de las creencias y, en este sentido, (b) constituye una distinción pero no de principio porque depende de (c), i.e., la distinción entre contenido conceptual y contenido no conceptual, de manera que es realmente (c) el que proporciona el criterio fuerte para la distinción entre los ES y los EI. ${ }^{6}$

Tal como se mencionó antes, una estrategia para discutir (b) sería argumentar que la red de creencias no está integrada de la manera en que Stich y otros creen. Stich (1978) parece decir que las creencias están sometidas a clausura bajo consecuencia lógica (flexible, esto es, bajo ciertas condiciones): si uno cree que $P$ entonces $Q$, y si cree que $P$, entonces podría llegar a creer que $Q$. La clausura no se sostiene si interviene un $\mathrm{ES}$, porque si se tiene una creencia condicional $C$ y un ES $S$ (que es el antecedente de $C$ ), no se llega a creer el consecuente del condicional. Sin embargo, hay ejemplos en los que las creencias tampoco se combinan de manera de dar lugar a otra nueva y esto no mostraría que alguna de ellas no sea una creencia. Con respecto a la otra estrategia mencionada, la de discutir en contra de que los ES no estén integrados inferencialmente con la red de creencias, la idea sería básicamente la misma, la razón por la cual no habría integración inferencial entre los ES y las creencias no se debe al carácter subdoxástico de alguna de las premisas. Richard (1994) muestra la plausibilidad de ambas estrategias a través de tres propuestas de interpretación de la noción de "promiscuidad inferencial".

\footnotetext{
${ }^{6}$ Davies rechaza la idea de que la modularidad fundamenta (b), esto es, que la modularidad sea la razón por la cual los ES no están inferencialmente integrados. Esta estrategia, simplemente, le permite rechazar una propuesta de fundamentación de (b) para defender su propia propuesta de fundamentación en (c).
} 
Richard propone entender la "promiscuidad inferencial" en términos de que los estados mentales tienen contenidos que determinan condiciones de verdad y relaciones de implicación y, en general, están bajo clausura de implicación lógica (si las condiciones son las adecuadas). Según esta definición, la combinación de la creencia de que si Twain duerme, entonces hay problemas, junto con la creencia de que Clemens duerme, no lleva a la creencia de que hay problemas, porque aunque Twain duerme y Clemens duerme tienen las mismas condiciones de verdad, el contenido es distinto ya que son las propiedades lógicas (sintácticas) las que determinan la identidad de contenido. De igual forma, los ES no comparten contenidos con las creencias, porque probablemente difieren en estilo de representación y, dado que la identidad de contenido depende (en parte) de relaciones lógicas y éstas de la sintaxis, las interacciones entre ambos tipos de estados estarían bloqueadas. ${ }^{7}$ Así:

Para que dos estados tengan un tercer estado como consecuencia lógica, tienen que tener contenidos relacionados adecuadamente. (Compárese: para que dos oraciones tengan una tercera oración como consecuencia lógica, tienen que tener una sintaxis relacionada adecuadamente.) Dado que las creencias y los estados subdoxásticos no comparten contenidos, no tienen consecuencias lógicas (significativas). (Compárese: dado que "Twain duerme" y "Si Clemens duerme, habrá problemas" no están adecuadamente relacionadas en contenido, no tienen consecuencias lógicas interesantes.) Así, que haya relaciones inferenciales relativamente empobrecidas entre creencias y estados subdoxásticos no muestra bajo la caracterización de promiscuidad en cuestión que las creencias y los estados subdoxásticos no son conjuntamente promiscuos. Considerando que no comparten contenidos no necesitan interactuar inferencialmente [...] para ser conjuntamente promiscuos, puesto que un conjunto de estados es promiscuo cuando sus miembros que tienen contenidos inferencialmente relacionados se combinan para producir nuevos estados. (Richard 1994, p. 306)

Hasta aquí la noción de inferencia que está en juego es la tradicional y, según esta noción, parece que no es cierto que la red de creencias sea promiscua en el sentido definido. Si, por otro lado, se entiende la noción de "patrones de inferencias" en términos de mecanismos causales, cualquier noción de "promiscuidad" parece aplicarse tanto a las creencias como a los ES. Richard propone dos

${ }^{7}$ La opción de que las creencias estén bajo clausura sólo por implicaciones de condiciones de verdad parece implausible, véase Richard 1994, pp. 305-306. 
maneras de entender esta última noción. La primera establece que "[una creencia-tipo] $b$ es promiscua con la colección de estados-tipo $S$ siempre que haya, para muchos de los miembros $b^{\prime}$ de $S$, algún subconjunto 'adecuado' $X$ de $S$ y un patrón de inferencia $I$ tal que $X, b \vdash, b^{\prime \prime}$ (p. 307). Según esta noción, nada impediría que los ES sean promiscuos con las creencias. Se podría pensar que cierto patrón de inferencia $I$ proyecta un $\operatorname{ES} S$ en una creencia $C$, que a su vez es proyectada por otro patrón inferencial $I^{\prime}$ a otra creencia $D^{\prime}$. De modo que podría ser posible agrupar los mecanismos causales de manera que den lugar a (inferir) un patrón (mecanismo causal) $I^{\prime \prime}$ que lleve de $S$ a $D^{\prime} .8$

La segunda manera de entender la noción de "promiscuidad", siguiendo la idea de Stich de que las relaciones inferenciales entre los ES son especializadas puesto que los mecanismos en los que intervienen son de propósito específico mientras que los mecanismos que se aplican a las creencias son de propósito general, establece que "[una creencia-tipo] $b$ es promiscua con la colección de estadostipo $S$ siempre que haya, para muchos de los miembros $b^{\prime}$ de $S$, algún subconjunto 'adecuado' $X$ de $S$ y un patrón de inferencia $I$ que es general para $S$ tal que $X, b \vdash, b^{\prime \prime}$ (p. 308). Según esta noción, no parece descabellado suponer que una regla general como el modus ponens, entendida en términos de un mecanismo causal que se aplica a las creencias, no se aplique también a los ES. Se podría dar el caso de que hubiera un mecanismo causal que cada vez que se tiene un ES $S$ (por ejemplo, expresado con la afirmación "Una oración con cierto análisis sintáctico $S$ ha sido emitida") causara un estado de creencia $C$ (por ejemplo, "Canadá ha sido invadida por Finlandia"), y sería plausible suponer que hay una instancia de un estado representacional $S / C$ que es de la forma Si $S$ entonces $C$ ("Si una oración con el análisis $S$ es emitida, entonces Canadá ha sido invadida por Finlandia"), de manera que el mecanismo causal llevaría al individuo a $C$ cuando está en $S / C$ y $S$.

Si contraejemplos como los de Richard resultan válidos, esto mostraría que una distinción fundamentada entre ES y EI no podría basarse en el criterio (b) de integración inferencial.

\footnotetext{
${ }^{8}$ Esto supone que los patrones de inferencias están bajo clausura de producto. Según Richard, se podría negar esta propiedad pero habría que justificar esto de manera que no sea arbitraria la restricción acerca de qué cuenta como un patrón de inferencia.
} 


\section{Conceptualización}

La idea de que tener una creencia requiere posesión de conceptos es una de las pocas cuestiones en las que concuerdan los filósofos. Un individuo no puede creer que $p$ o tener el pensamiento de que $p$, donde $p$ es, por ejemplo, el agua es inodora, sin tener los conceptos involucrados en la proposición (AGUA e INODORA). Según Evans (1981a), el que una creencia esté al servicio de distintos propósitos supone que el individuo es sensible al lugar que ocupa esa creencia en su red de creencias; esto es, es sensible a su potencial inferencial: las creencias de las cuales se infiere esa creencia y las que se pueden inferir a partir de ella. Esto, a su vez, supone que las creencias (pensamientos, en general) son estados estructurados en el sentido de ser un complejo de habilidades, por ejemplo, creer que $F a$ consiste en el ejercicio de dos habilidades, la correspondiente a $a$ y a $F$.

Así, ser sensible al potencial inferencial de la creencia de que $a$ es $F$ depende de la sensibilidad al potencial inferencial de otras creencias en las que están involucrados a y $F$ (el pensamiento $F a$ sería la intersección de dos series, la de $F a, F b, \ldots$, y la de $F a$, $G a, \ldots)$. Si un sujeto tiene los pensamientos estructurados de que $a$ es $F$ y $b$ es $G$, no habría en principio ninguna barrera conceptual para tener el pensamiento de que $a$ es $G$ o que $b$ es $F$. En esta capacidad de ejercitar habilidades cognitivas consiste justamente la posesión de conceptos, de manera que tener una creencia depende de habilidades conceptuales estructuradas:

Así, si a un sujeto se le atribuye el pensamiento de que $a$ es $F$, entonces debe tener los recursos conceptuales para tener el pensamiento de que $a$ es $G$, para cualquier propiedad de ser $G$ de la cual tiene una concepción. Ésta es la condición que llamo "el requisito de generalidad". (Evans 1982, p. 104) $)^{9}$

${ }^{9} \mathrm{Si}$ bien la polémica entre los conceptualistas y los no conceptualistas parece encuadrarse más cómodamente en una concepción de los conceptos en cuanto particulares mentales (dado que, con respecto al contenido de los estados mentales, lo que está en juego es si los conceptos son sus "constituyentes" mentales), introduzco el requisito de generalidad en términos de habilidades para respetar la formulación original de Evans que toma Davies (y, en consecuencia, para respetar los términos en los que el debate acerca del contenido no conceptual se planteó en sus comienzos, cfr. Heck en prensa). No creo que nada de lo que sigue dependa de esta manera de presentar el requisito, pero, si ello fuera así, entonces se podría entender esta caracterización o, al menos, coincido con Davies (1998) en hacerlo así, en el sentido de que estas habilidades cognitivas tienen una base categorial (un mecanismo causal) subyacente. Agradezco a uno de los árbitros la posibilidad de aclarar este punto y el de la nota siguiente. 
Siguiendo la propuesta de Evans (1982, p. 104, n. 22) de que el requisito de generalidad se aplica al ámbito intencional pero no a los estados informacionales, Davies (1989) considera que este requisito provee el criterio buscado para una distinción fundamentada, en el sentido de que explicita parte de lo que está involucrado en la posesión de conceptos, de manera que la distinción entre dos tipos de contenido carga todo el peso de la distinción doxástico-subdoxástico: los EI tienen contenido conceptual, a diferencia de los ES, cuyo contenido es no conceptual. ${ }^{10}$ Dado que poseer un contenido intencional (de que $a$ es $F$ ) es ipso facto cumplir con el requisito de generalidad (ejercitar el dominio del concepto de ser $F$ ), el requisito de generalidad es, parcialmente, constitutivo de la noción de "contenido conceptual". Los conceptos son los constituyentes de los pensamientos, de modo que tener un pensamiento es ipso facto tener los conceptos que lo constituyen. En cambio, no se requiere que el sujeto ejercite habilidades conceptuales particulares para estar en un estado informacional. Desde esta perspectiva, ambos tipos de contenido son representacionales, en el sentido de que el mundo está representado de cierta manera, pero en el caso del no conceptual no se ejercitan habilidades conceptuales.

Podría darse el caso de que los ES de un sistema de procesamiento de información cumplieran el requisito de generalidad, pero su cumplimiento sería contingente, dado que la posesión de conceptos no es una condición esencial para el contenido de los ES. De ahí que los EI y los ES en conjunto no satisfacen el requisito de generalidad:

Un pensador podría tener el pensamiento de que a es $F$, y estar en un estado subdoxástico que tiene el contenido de que $b$ es $G$. Sin embargo, podría ser que el pensador no pudiera tener el pensamiento de que $a$ es $G$-porque carece del concepto de ser $G$-; y podría ser que no haya un estado subdoxástico real o posible cuyo contenido sea que $a$ es $G$, porque el sistema que procesa la información de que $b$ es $G$ simplemente no contiene información acerca del objeto $a$. (Davies 1989, p. 148)

\footnotetext{
${ }^{10}$ Estoy de acuerdo con Heck (2000, en prensa) en que el no conceptualismo que defiende Evans (y el que suscribe Davies en su defensa de (c)) es el de contenido (y no, simplemente, el no conceptualismo de estado). El requisito de generalidad provee una manera de entender la idea de que las creencias, los pensamientos y similares, poseen contenido conceptual en el sentido de que los pensamientos están articulados conceptualmente. En este sentido, el contenido de los EI es de un tipo diferente del de los ES.
} 
Una estrategia para discutir el criterio (c) de conceptualización sería mostrar que el requisito de generalidad tiene contraejemplos. ${ }^{11} \mathrm{Si}$ esto es así y si se suponía que el requisito explicitaba (parte de) lo que está involucrado en la noción de "contenido conceptual", entonces nos quedaríamos con una noción de "contenido conceptual" vacía. Y en tanto que la noción de "contenido no conceptual" es derivada de la de "contenido conceptual", no habría una distinción fundamentada entre ambas nociones. Esta estrategia no estaría mal, pero no parece muy promisoria. Lo que podría hacer un defensor de la distinción que no avale el requisito es ofrecer un enfoque para dar cuenta de en qué consiste tener los recursos (o capacidades) conceptuales para tener un pensamiento particular $F a$ (o como suele especificarse esto: en qué consiste que un individuo esté en condiciones de conocer lo que hace que cualquier objeto arbitrario sea $F$ o, lo que es lo mismo, que cierto pensamiento $F a$ sea verdadero) que, por supuesto, no esté ligado a (no suponga) el requisito de generalidad. Y esto parece difícil de lograr. ${ }^{12}$

Más allá de las dificultades que pueda haber para dar sustancia a la noción de "contenido conceptual", es innegable que la distinción conceptual/no conceptual parece recoger una intuición muy fuerte acerca de las capacidades cognitivas humanas. La intuición afirma que la especie humana comparte ciertas capacidades cognitivas (inferiores) con otras especies, y algunas (las superiores) son sólo propias de los humanos. Si hay algo que nos distingue claramente del resto de los animales son nuestras capacidades lingüísticas y dado que, en general, se cree que el lenguaje expresa el pensamiento, las teorías acerca del pensamiento le han atribuido a éste propiedades similares a las del lenguaje. De modo que, no resulta extraño pensar que así como dominar un lenguaje requiere dominar términos, y dado que los conceptos son los constituyentes del pensamiento, todo contenido intencional de nuestros pensamientos, en general, es contenido conceptual. Por otro lado, si hay algo que nos equipara con el resto de las especies es la capacidad de procesar información. Una de las motivaciones para la postulación de contenido no conceptual es la

${ }^{11}$ Richard (1994, pp. 310-315) presenta un contraejemplo al requisito y también objeta la versión del mismo de Davies 1989. Véase también Travis 1994, que intenta mostrar que el requisito no se sostiene para el pensamiento proposicional.

${ }^{12}$ Cfr. la objeción de Richard 1994 al intento de Peacocke 1992. Por supuesto que en la literatura sobre el tema hay varias definiciones de "contenido conceptual" (y "no conceptual"), la cuestión es que esas definiciones requieren un enfoque acerca de la posesión de conceptos y es, justamente, este enfoque el que muy probablemente apele a una u otra variante del requisito de generalidad. 
idea de que hay estados de procesamiento de la información que compartimos con otras especies y para los cuales estar en ellos no requiere que la criatura domine conceptos. ${ }^{13}$

Supongamos que quisiéramos respetar la intuición fuerte de una distinción entre contenido conceptual y contenido no conceptual (debido a sus legítimas motivaciones evolutivas, ontogenéticas, y demás), y que aceptamos que, en el dominio intencional, el contenido de los estados mentales está conceptualizado. Aun así, la característica de poseer contenido no conceptual no clasifica a un estado como subdoxástico.

Si bien Davies no ofrece, en su artículo, ninguna formulación explícita acerca de en qué consiste que un criterio establezca una distinción de principio entre los EI y los ES, se pueden extraer de su texto dos versiones, una fuerte y otra débil. La fuerte diría que un criterio establece una distinción de principio cuando es una condición necesaria y suficiente (o, menos fuerte, cuando sólo es una condición suficiente) para que un estado sea o bien doxástico o bien subdoxástico; la débil establecería el principio de que el criterio no clasifique como ES a estados que intuitivamente son bastante diferentes de los estados psicológicos que tomaríamos prima facie como ejemplos de ES. Ambas versiones pueden verse funcionando en el tratamiento que hace Davies del criterio de accesibilidad a la conciencia, que he abordado en la sección 1 . Davies no habla explícitamente en términos de condición necesaria (sí de suficiente, por ejemplo: "Así, sería mejor que la inaccesibilidad a la conciencia no fuera una condición suficiente para que un estado sea subdoxástico"; 1989, p. 136), pero está claro que la razón 3, también vista en la sección 1, establece que (a) no es una condición necesaria. La versión débil apela a las intuiciones de los filósofos y se extrae de pasajes como el siguiente: "Pero estos estados [creencias inconscientes] son intuitivamente

${ }^{13}$ Hay otras motivaciones relacionadas con las experiencias perceptuales. En particular, la idea de que el contenido experiencial es de grano más fino que los conceptos perceptuales (podemos discriminar tonos de colores para los cuales podríamos no tener conceptos de color) y que el contenido experiencial es independiente de las capacidades conceptuales (en el caso de las ilusiones visuales, éstas persisten aun no creyendo lo que se ve). Véase McDowell 1994 (cap. 3) para una discusión de estas motivaciones. También hay motivaciones relacionadas con elementos demostrativos y deícticos contenidos, de manera explícita o implícita, en los estados representacionales. Véanse, entre otros, Perry 1979, Peacocke 1986 y Cussins 1990. Por otro lado, véase Speaks 2005, que intenta mostrar que estos argumentos clásicos a favor del contenido no conceptual no sólo no dan apoyo al no conceptualismo de contenido, sino que tampoco permiten sostener un no conceptualismo de estado. 
bastante diferentes de los estados psicológicos cognitivos que tomaríamos como ejemplos prima facie de subdoxasticidad" (1989, p. 136).

Si la distinción entre el contenido conceptual y el contenido no conceptual estableciera realmente una distinción de principio, en el sentido fuerte, entre ES y EI, la sola presencia de contenido conceptual haría (sería una condición suficiente para) que un estado clasificara en el ámbito intencional, y la sola presencia de contenido no conceptual haría que cierto estado clasificara como subdoxástico. Sin embargo, si bien por mor del argumento la primera condición parece cumplirse, la segunda no. Hay quienes consideran que las experiencias perceptuales tienen contenido no conceptual, entre ellos, el propio Davies (1995). Dado que el contenido no conceptual sería la marca de lo subdoxástico, tendríamos la consecuencia antiintuitiva (y contraria a la evidencia psicológica) de que las experiencias perceptuales serían ES. Sin embargo, esto no sólo no es así bajo ninguna concepción disponible de experiencia perceptual, sino tampoco en el sentido débil de Davies.

Los ejemplos de estados perceptuales de procesamiento de la información que figuran en la literatura sobre la visión se refieren a los estados perceptuales del procesamiento visual temprano, que no constituyen por sí mismos experiencias perceptuales. Nadie, hasta donde sé, considera que las experiencias perceptuales sean meramente procesamiento de información. Evans (1982, p. 157), por dar sólo un ejemplo, defiende esta distinción cuando afirma: "he considerado el contenido no conceptual de los estados informacionales perceptuales. Estos estados no son ipso facto experiencias perceptuales, esto es, estados de un sujeto consciente". Hay razones conceptuales y empíricas que contribuyen a esta distinción. Por un lado, el hecho de que las experiencias, a diferencia de los ES, son estados conscientes, para los cuales además de los estados de información perceptual se requiere la interacción de los mismos con otros subsistemas como la memoria (o experiencia previa), la atención, etc. Pero si no se quiere que intervenga el rasgo (a), hay argumentos que apelan a la evolución sosteniendo que es razonable suponer que organismos con conexiones de inputs perceptuales-outputs conductuales han surcado la tierra antes que organismos con experiencias conscientes. Por otro lado, hay casos de pacientes con daño cerebral que tienen, por ejemplo, alguna forma de agnosia, esto es, no reconocen objetos familiares a pesar de que los mecanismos sensoriales están intactos, o de "ceguera percep- 
tual", esto es, procesan la información perceptual pero no tienen la "experiencia perceptual". 14

Por otro lado, las experiencias perceptuales tienen características que no poseen los ES; en particular, están ligadas a la conciencia fenoménica. Tienen un componente fenoménico, que consiste en cómo es estar en esa experiencia, el cual no acompaña a ningún estado de procesamiento de la información. No hay algo así como qué se siente estar en el ES que consiste en un cierto arreglo de rasgos léxicos (ni tendría sentido que lo hubiera, si es que hay algo como qué se siente estar en un estado mental en particular). De manera que si el criterio (c) de conceptualización/no conceptualización del contenido, entendido ahora en el sentido débil, permite que las experiencias perceptuales (estados que son bastantes diferentes de los estados cognitivos que tomaríamos prima facie como estados de procesamiento de la información) queden clasificadas como ES, es porque el criterio no funciona.

Se podría pensar que la clasificación de Davies no pretende ser exhaustiva en el sentido de abarcar a todos los tipos de estados mentales, sino sólo discriminar entre actitudes proposicionales y estados de procesamiento de la información. De modo que las experiencias perceptuales podrían caer del lado subdoxástico sin que esto constituyera una objeción a (c), puesto que lo único que haría que (c) no funcionara como criterio, según la versión débil, es si ciertos estados de actitud proposicional cayeran del lado subdoxástico. ${ }^{15}$ Dos respuestas breves. En primer lugar, la formulación implícita de lo que denominé el sentido débil en el que un criterio establece una "distinción de principio" parece decir que si hay estados que son bastantes diferentes de los estados cognitivos que tomaríamos prima facie como estados de procesamiento de la información, los cuales son clasificados como ES según un criterio determinado, entonces ese criterio no funciona. Si la intención es descartar sólo las actitudes proposicionales, este principio sería sumamente trivial porque diría que un criterio que no permite distinguir entre actitudes proposicionales y ES no funciona. Si no se quiere que el principio intuitivo sea trivial, debería admitirse la posibilidad de que si bajo ese criterio cae cualquier estado mental bastante diferente de los estados mentales que tomaríamos prima facie como estados de procesamiento de la

${ }^{14}$ Por ejemplo, en los casos de blindsight (Weiskrantz 1986), en los que la destrucción de la corteza visual primaria (V1) lleva a una ceguera en el campo visual opuesto al hemisferio dañado, los pacientes pueden detectar estímulos de los cuales no son conscientes visualmente.

${ }^{15}$ Agradezco a Diana Pérez esta observación. 
información, entonces ese criterio no funciona. En segundo lugar, si le concedemos a Davies que la clasificación no pretende ser exhaustiva, entonces el criterio de contenido no conceptual no vulnera el sentido débil (porque simplemente el sentido débil ya no hace ningún trabajo, se lo ha vaciado), pero sigue sin ser una condición suficiente para que un estado mental clasifique como subdoxástico. ${ }^{16}$

\section{Una distinción alternativa posible}

Este trabajo podría haber concluido en la sección anterior. El propósito del mismo era mostrar que los criterios (a)-(c) no son buenos criterios para establecer una distinción entre EI y ES y que, por ende, esta distinción no sólo se desdibuja, sino que nos quedamos sin poder hacer justicia a ciertas intuiciones filosóficas acerca de que habría distinciones entre tipos de estados mentales que serían pertinentes para la ciencia cognitiva; después de todo este objetivo motivó, en primer lugar, la postulación de la distinción doxástico-subdoxástico. Sin embargo, no puedo dejar de decir algo acerca de una posible vía para rescatar estas intuiciones, que es, a la vez, sólida e interesante para la ciencia cognitiva; aunque, por razones de extensión, aquí no podré argumentar en favor de su solidez, sino sólo mostrar que funciona allí donde la distinción doxástico-subdoxástico, por sí misma, no lo hace; y sólo podré mencionar, sin desarrollarlos, los motivos por los cuales esta vía es pertinente o interesante para la ciencia cognitiva.

En verdad, el rasgo fundamental que distingue a estados con contenido no conceptual, como los del procesamiento de la información y las experiencias perceptuales, es que los primeros están en el nivel subpersonal, mientras que los segundos están en el nivel personal. De manera que el defensor de (c), y también los defensores del resto de los criterios, tal como se verá más adelante, tendrían que apelar a un criterio ulterior basado en la distinción personalsubpersonal. Diré algo sobre la distinción, aunque insisto en que mi propósito en este trabajo no es constructivo sino negativo, esto es, criticar los fundamentos de la distinción doxástico-subdoxástico. En otro lugar me he ocupado de la cuestión de cómo la distinción personal-subpersonal es más adecuada para recoger una diferencia

\footnotetext{
${ }^{16}$ Parecería muy rara una condición suficiente que dijera que tener contenido no conceptual es suficiente para que un estado mental sea subdoxástico, a menos que ese estado mental sea $X$, por ejemplo, una experiencia perceptual, o $Y$ o $Z$ o lo que fuere que tuviera contenido no conceptual además de los ES.
} 
real e interesante, para la psicología cognitiva, entre diferentes clases de estados mentales. ${ }^{17}$

Parece haber una distinción, por ejemplo, entre ciertos estados internos del sistema de procesamiento visual, como las imágenes retinales que representan valores de intensidad, y estados mentales, como por ejemplo, ver una silla. Mientras que los primeros son procesados por subsistemas de la persona y, en ese sentido, son estados subpersonales de procesamiento de información (o atribuibles a subsistemas cognitivos de la persona), los segundos no son estados que le acaezcan a alguna parte de la persona, sino que son algo que ella, en cuanto tal, hace y, en ese sentido, son estados perceptuales de (o atribuibles a) la totalidad de la persona. La distinción personal-subpersonal recoge tanto diferentes dominios discursivos cognitivos como diferentes fenómenos cognitivos (también recoge dominios discursivos y fenómenos físicos, pero véase lo que afirmo más adelante). En términos discursivos, el vocabulario que se utiliza en las descripciones del nivel personal es el de las categorías de la psicología de sentido común. La explicación intencional típica de la psicología de sentido común se basa en el principio de que si una persona desea $Y$ y cree que puede obtener $Y$ haciendo $X$, entonces ceteris paribus hará $X$. Esta explicación tiene como objetivo hacer inteligible el comportamiento de las personas a la luz de un patrón más amplio de actividad racional.

Por otra parte, habría dos tipos de descripciones y explicaciones en el nivel subpersonal: las neurofisiológicas y las de procesamiento de la información. La primera emplea vocabulario de las neurociencias, mientras que la segunda utiliza vocabulario de la psicología de procesamiento de la información. Si la distinción personal-subpersonal se concibe como una distinción intracognitiva, lo que interesa, entonces, es el último tipo de dominio discursivo y de fenómenos. De manera que "subpersonal" hace referencia al funcionamiento del cerebro, pero no en cuanto tal, sino como sistema de procesamiento de la información. La explicación subpersonal típica es el análisis funcional (Cummins 1983). El primer paso del análisis funcional consiste en la descomposición de la capacidad cognitiva $P$ en subcapacidades, y el segundo, en dar cuenta de la instanciación en el sistema cognitivo $S$ del análisis de $P$. De modo que si $S$ posee los componentes organizados de la manera especificada en el análisis, entonces $S$ posee

\footnotetext{
${ }^{17}$ Argumento a favor de esta sugerencia en Skidelsky 2003. Para la formulación original de la distinción, véase Dennett 1969; para una reflexión acerca de la distinción denettiana, véase Skidelsky y Pérez 2005; para aplicaciones actuales, véase Bermúdez y Elton 2000.
} 
$P$. La explicación funcional concluye cuando el sistema $S$ ejecuta el programa especificado por el análisis ( $S$ tiene que tener la estructura necesaria para cumplir las instrucciones del programa). En este sentido, los estados subpersonales figuran en explicaciones acerca de cómo las personas tienen ciertas capacidades subpersonales y personales. ${ }^{18}$

Está ampliamente aceptado, dada esta caracterización mínima de la distinción, que las actitudes proposicionales y las experiencias son estados personales, o atribuibles a la persona, y no a ningún subsistema de la persona. Son las personas, en cuanto sistemas cognitivos globales quienes creen, desean, y similares, y quienes experimentan dolor y demás. En cambio, los estados de procesamiento de la información son estados de (o atribuibles a) subsistemas de las personas, como los estados del procesamiento lingüístico temprano (que construye descripciones estructurales de las expresiones lingüísticas a partir de elecciones léxicas) y el procesamiento primario de la visión (que construye una representación tridimensional de objetos a partir de las imágenes retinales). Éstos son subsistemas cognitivos, cuyos outputs actúan como inputs para otros subsistemas que, eventualmente, dan lugar a estados personales. Por ejemplo, en el caso del lenguaje, las descripciones estructurales que son el producto de la lengua-I son el output de un subsistema muy específico de la arquitectura mental. Estas representaciones tienen que ser interpretadas por los sistemas de ejecución lingüística: el articulatorio-perceptual y el conceptualintencional. La interacción con estos sistemas de ejecución, que a su vez se relacionan con los intereses, los propósitos y el conocimiento general de los hablantes, permite que las representaciones lingüísticas así enriquecidas y transformadas participen de la comunicación lingüística, el pensamiento y la acción en general, todos fenómenos del nivel personal.

Teniendo en cuenta esta distinción, se podrían manejar los casos (si los hubiera) que hemos visto y que los criterios propuestos no permitían: el de las creencias inconscientes (estados personales), la promiscuidad inferencial entre ES (estados subpersonales), y la cuestión del contenido no conceptual, rasgo compartido por los ES (estados subpersonales) y las experiencias perceptuales (estados personales).

El criterio (a) de accesibilidad a la conciencia, por sí mismo, no permite distinguir entre estados doxásticos y de procesamiento de la información, porque, entre otros problemas, hemos visto que cabría

\footnotetext{
${ }^{18}$ Para un mayor desarrollo de las características mencionadas y críticas a propuestas recientes de relación entre ambos niveles, véase Skidelsky 2006.
} 
la posibilidad de que hubiera creencias inconscientes y con ello habría EI que, bajo el criterio (a), clasificarían como subdoxásticos. Si tomamos en cuenta la distinción personal-subpersonal, las creencias inconscientes no se volverían estados subdoxásticos por ser inconscientes, dado que las creencias no son estados de los subsistemas de las personas, sino estados personales, esto es, de (o atribuibles a) la persona. Esto es así porque, entre otras razones, los estados de creencia (sean conscientes o inconscientes) satisfacen ciertos requisitos mencionados antes para ser considerados estados personales, como el hecho de que son expresados en el vocabulario conformado por las categorías intencionales de la psicología de sentido común, y son utilizados en las atribuciones y explicaciones intencionales cuyo fin es hacer inteligible la conducta de los individuos, en cuanto agentes racionales que actúan en virtud de esos estados (sean conscientes o no).

El criterio (b) tampoco permite, por sí mismo, la distinción entre estados doxásticos y subdoxásticos porque o bien no hay tanta integración inferencial en el nivel intencional como se piensa, o bien parece haber integración inferencial en ambos ámbitos. Incluso, dado que puede haber tanto estados personales como subpersonales integrados, en mayor o menor grado, inferencialmente (y, probablemente, entre sí), el criterio (b) de integración inferencial no parece ser un criterio delimitador de principio. Sin embargo, sería posible diferenciar el tipo de estados que participan en los procesos inferenciales aplicando la distinción personal-subpersonal. Desde la perspectiva de la ciencia cognitiva computacional, todo sistema cognitivo procesa información y este procesamiento puede verse como transformaciones de instancias de oraciones; en este sentido, toda capacidad cognitiva puede caracterizarse como una capacidad inferencial. Los sistemas cognitivos instancian funciones cognitivas cuyos argumentos y valores están relacionados epistémicamente; esto es, dado ciertos inputs, el output es racional (en el sentido de "convincente") a la luz de aquellos (Cummins 1983, cap. 3). ${ }^{19}$ Así, los sistemas cognitivos son mecanismos inferenciales que toman argumentos y valores, y los relacionan como se relacionan las premisas con la conclusión; los inputs y los outputs tienen una interpretación proposicional, ya que las proposiciones son el tipo de elementos que participan de procesos inferenciales. ${ }^{20} \mathrm{En}$

\footnotetext{
${ }^{19}$ Una secuencia es convincente (cogent) cuando cada parte lleva convincentemente a la siguiente, de manera que cada consecuencia es la continuación natural e inevitable de su antecedente.

${ }^{20}$ Que haya una interpretación de los inputs y los outputs en términos proposicionales no implica ningún compromiso ontológico con símbolos en la cabeza.
} 
general, tanto para mecanismos subpersonales — por ejemplo, el procesamiento visual temprano o el análisis lingüístico-, como para los personales — por ejemplo, el pensamiento-, hay una descripción en términos inferenciales. ${ }^{21}$ Lo que permitiría distinguir a qué tipo psicológico pertenece un estado integrado inferencialmente sería su clasificación como estado personal o subpersonal, esto es, entre otras cosas, el vocabulario que se utilice para su descripción (si el de la psicología de sentido común o el de la psicología de procesamiento de la información), el tipo de explicación psicológica en el que participa (las intencionales de la psicología de sentido común o las de análisis funcional de la psicología de procesamiento de la información), etcétera.

El criterio (c), esto es, que el contenido sea conceptual o no conceptual, por sí mismo tampoco establece la distinción, porque hemos visto que poseer contenido no conceptual no es una condición suficiente para que un estado sea de procesamiento de la información (el caso de las experiencias), y al mismo tiempo, clasifica estados que prima facie no consideraríamos de procesamiento de la información (las experiencias). En cambio, la distinción personal-subpersonal clasificaría los estados con contenido no conceptual, esto es, las experiencias y los estados de procesamiento de la información, en ámbitos distintos, el personal y el subpersonal, respectivamente. Esto es así por las razones que he mencionado acerca del vocabulario, y el objetivo y el tipo de explicación que se utilizan en ambos niveles.

En realidad, creo que la distinción doxástico-subdoxástico parece ser poco interesante para la psicología cognitiva (subrayo, especialmente, "para la psicología cognitiva"), en la medida en que, por un lado, tiene los problemas que ya hemos visto - esto es, ninguno de los criterios propuestos, por separado, y en conjunto, funciona$\mathrm{y}$, por el otro, la distinción personal-subpersonal parece abarcarla y dar respuesta a la cuestión de tener una distinción sólida (como he mencionado, no es mi objetivo en este trabajo argumentar en favor de esta solidez) e interesante, para la psicología cognitiva, entre diferentes clases de estados mentales. ${ }^{22}$

\footnotetext{
${ }^{21}$ Nótese, además, que tanto Marr 1982 como Chomsky 1980b describen el procesamiento de las respectivas capacidades subpersonales como inferencial.

${ }^{22}$ Digo "abarcarla" y no "reemplazarla" porque creo que los criterios (a)-(c) se conservan, con ciertas modificaciones, en la distinción personal-subpersonal (junto con los mencionados acerca del criterio del sujeto de la atribución; i.e., persona en oposición a subsistemas de la persona, el vocabulario, y el tipo y objetivo de la explicación psicológica. Véase Skidelsky (en prensa) para una propuesta de un criterio de distinción basado en el contenido de los estados subpersonales). En este
} 
Recordemos que, según Stich (1978), la fundamentación de la distinción intuitiva entre estados doxásticos y subdoxásticos tenía como objetivo marcar un límite psicológico entre dos tipos de estados mentales que fuera interesante para la psicología cognitiva. El interés radica en evitar líneas de investigación que lleven al fracaso - por ejemplo, desarrollar modelos inferenciales para los ES- y abordar cuestiones fructíferas - por ejemplo, si hay un mecanismo común para la integración inferencial y la accesibilidad a la conciencia-. $\mathrm{Si}$, tal como hemos visto, la fundamentación de los criterios sobre los que se basa la distinción doxástico-subdoxástico no permite marcar este límite psicológico, el interés para la psicología cognitiva se desvanece. Por ejemplo, si no es cierto que los ES están integrados inferencialmente de manera pobre, entonces no es aconsejable evitar el desarrollo de modelos inferenciales para esos estados, y si resulta que hay estados doxásticos inconscientes, entonces no es el caso que la integración inferencial y la accesibilidad a la conciencia sean rasgos que vayan juntos.

Así, mientras que la distinción doxástico-subdoxástico pierde interés para la ciencia cognitiva, la distinción personal-subpersonal no sólo lo adquiere porque permite conservar la intuición filosófica de que habría una distinción real entre tipos de estados mentales, sino que cumple un papel crucial para cuestiones cognitivas clave tales como: cuál es la tarea de la psicología cognitiva (si explicar el funcionamiento de los procesos subpersonales y/o dar cuenta también del ámbito personal), qué tipo de contenido mental poseen los estados subpersonales a diferencia de los personales (si conceptual o no conceptual, estrecho o amplio, etc.) y la cuestión de cómo se relacionan las partes subpersonales del sistema cognitivo global para dar lugar a la integración de los subsistemas y, en última instancia, al comportamiento humano.

sentido, la relación entre las distinciones no es de paralelismo; tampoco quiero decir que la distinción personal-subpersonal resuelva todos los problemas para los cuales se propuso la distinción doxástico-subdoxástico (agradezco a uno de los árbitros la posibilidad de aclarar este punto). Supongo que esta última también tiene fines epistemológicos a los cuales la distinción personal-subpersonal no tendría nada que aportar dado que su formulación surgió con propósitos puramente psicológicos (más exactamente, relacionados con la explicación psicológica, cfr. Dennett 1969). Así, en este ámbito, el de la psicología cognitiva, y en función de la solución de los problemas que hemos visto y de los intereses psicológicos para los cuales se planteó, sostengo que la distinción personal-subpersonal funciona allí donde no funciona la distinción doxástico-subdoxástico. 


\section{Conclusión}

La discusión que Davies (1989) realiza de los criterios (a)-(c) tenía como objetivo explorar las diferencias intuitivas entre los ES y los EI con el fin de ver si se podían dar argumentos en favor de una distinción fundamentada. Si se hace una lectura radical de Davies 1989, su objetivo sería concluir que es (c), el criterio de conceptualización, el que carga con todo el peso de la distinción: puesto que el criterio (a) de accesibilidad a la conciencia y el (b) de integración inferencial no se sostienen por sí mismos, requieren ser fundamentados en (c). ${ }^{23}$ Este criterio fundamenta (a) porque si se quiere que (a) haga el trabajo para el cual se lo formuló debería refinarse la noción preteórica de "accesibilidad a la conciencia" de manera que se entienda como disponibilidad de los contenidos para el pensamiento y esto, por supuesto, depende de que el contenido esté conceptualizado (así, un estado es subdoxástico si estar en ese estado no es ipso facto tener el contenido disponible como contenido del pensamiento). También (c) fundamenta (b) porque el hecho de que el dominio de las actitudes proposicionales y los ES en conjunto no cumplan el requisito de generalidad es la consecuencia de que el contenido de los ES no es conceptual.

Pero si la búsqueda de un criterio que pueda proveer una distinción de principio quiere decir la búsqueda de una condición (necesaria y) suficiente para que un estado cuente como un estado de procesamiento de la información, el contenido no conceptual no califica, pues no es una condición suficiente, ya que las experiencias tienen contenido no conceptual y, sin embargo, no son estados de procesamiento de la información. Por otro lado, si la búsqueda de un criterio que pueda proveer una distinción de principio quiere decir que el criterio no clasifique como ES a estados que intuitivamente son bastante diferentes de los estados psicológicos que tomaríamos prima facie como ejemplos de ES, el contenido no conceptual no lo cumple, porque clasifica a las experiencias como estados de procesamiento de la información.

Ahora bien, si se hace una lectura menos radical, la idea es que (c) se suma a (a) y (b) en pos de una distinción fundamentada. No obstante, en este caso se requeriría, además, la distinción personalsubpersonal que, al discriminar entre aquellos estados que se atribuyen a la persona en su totalidad y aquellos que se atribuyen a los

${ }^{23}$ La intención de Davies parece ser la radical, véase Davies 1989, p. 148, y 1995, p. 376 . 
subsistemas de la persona, permitiría resolver algunos de esos problemas, que hemos visto, que esos criterios no podían manejar. En parte, creo que por este motivo la distinción doxástico-subdoxástico ha sido absorbida paulatinamente en la psicología cognitiva y, en general, en las ciencias cognitivas, por la distinción más abarcadora personal-subpersonal. ${ }^{24}$

\section{BIBLIOGRAFÍA}

Bermúdez, J.L. y M. Elton (comps.), 2000, Personal and Subpersonal: Essays on Psychological Explanation (special Issue), Philosophical Explorations, vol. 3, pp. 63-82.

Block, N., 1995, "On a Confusion about a Function of Consciousness", Behavioral and Brain Sciences, vol. 18, pp. 227-287.

Boden, M. (comp.), 1990, The Philosophy of Artificial Intelligence, Oxford University Press, Oxford.

Carruthers, P. y J. Boucher, 1998, Language and Thought, Cambridge University Press, Cambridge.

Chomsky, N., 2005, "Three Factors in Language Design", Linguistic Inquiry, vol. 36, no. 1, pp. 1-22.

- 2000, New Horizons in the Study of Language and Mind, MIT Press, Cambridge, Mass.

—_, 1995a, The Minimalist Program, MIT Press, Cambridge, Mass.

_- 1995b, "Language and Nature", Mind, vol. 104, no. 413, pp. 1-61.

_- 1994, "Naturalism and Dualism in the Study of Language and Mind", International Journal of Philosophical Studies, vol. 2, pp. 181200. (Reimpreso en Chomsky 2000, pp. 75-105.)

, 1986, Knowledge of Language, Praeger, Nueva York. [Versión en castellano: El conocimiento del lenguaje, trad. Eduardo Bustos Guadaño, Alianza, Madrid, 1989.]

——, 1980a, Rules and Representations, Blackwell, Oxford. [Versión en castellano: Reglas y representaciones, trad. Stephen A. Bastien, Fondo de Cultura Económica, México, 1983.]

${ }^{24}$ Una primera versión de este trabajo fue realizada en una estancia en el Center for Cognitive Science de Rutgers University en 2002. Agradezco muy especialmente a su director en ese momento, Ernie Lepore, el enriquecimiento académico del que he disfrutado en ese centro de excelencia. Versiones posteriores han sido presentadas durante 2004 en el Grupo de Acción Filosófica y en el VII Coloquio Internacional Bariloche de Filosofía. Agradezco a los participantes de estos encuentros, en particular, a Javier Castro Albano, Eduardo Barrio, Eleonora Orlando, Federico Penelas, y Diana Pérez por sus valiosas observaciones, y a Julia Vergara y Valeria Valiño por sus réplicas escritas al trabajo. La versión final se realizó durante 2004-2005 en una estancia posdoctoral en el Department of Philosophy de New York University gracias a un subsidio de la Fundación Antorchas. 
Chomsky, N., 1980b, "Rules and Representations", Behavioral and Brain Sciences, vol. 3, pp. 1-61.

- 1965, Aspects of the Theory of Syntax, MIT Press, Cambridge, Mass. [Versión en castellano: Aspectos de la teoría de la sintaxis, trad. Carlos Peregrín Otero, Aguilar, Madrid, 1970.]

Cummins, R., 1983, The Nature of Psychological Explanation, MIT Press, Cambridge, Mass.

Cussins, A., 1990, "The Connectionist Construction of Concepts", en Boden 1990, pp. 368-440.

Davies, M., 1998, "Language, Thought and the Language of Thought (Aunty's Own Argument Revisited)" en Carruthers y Boucher, pp. 226247.

- 1995, "Reply: Consciousness and the Varieties of Aboutness" en MacDonald y MacDonald 1995, pp. 356-392.

— , 1989, “Tacit Knowledge and Subdoxastic States", en George 1989, pp. 131-152.

— , 1986, "Tacit Knowledge and the Structure of Thought and Language", en Travis 1986, pp. 127-158.

Dennett, D., 1969, Content and Consciousness, Routledge and Kegan Paul, Londres.

Evans, G., 1982, The Varieties of Reference, Clarendon Press, Oxford.

- 1981a, Collected Papers, Clarendon Press, Oxford.

_- 1981b, "Semantic Theory and Tacit Knowledge", en Evans 1981a, pp. 322-342.

Fodor, J., 1983, The Modularity of Mind, MIT Press, Cambridge, Mass. [Versión en castellano: La modularidad de la mente, trad. J.M. Igoa, Morata, Madrid, 1986.]

George, A. (comp.), 1989, Reflections on Chomsky, Blackwell, Oxford.

Guttenplan, S. (comp.), 1994, A Companion to the Philosophy of Mind, Blackwell, Oxford.

Heck, R., 2000, "Nonconceptual Content and the 'Space of Reasons" ", The Philosophical Review, vol. 104, no. 4, pp. 483-523.

—_, en prensa, “Are There Different Kinds of Content?”, en McLaughlin y Cohen en prensa.

MacDonald, C. y G. MacDonald (comps.), 1995, Philosophy of Psychology, Blackwell, Oxford.

Marr, D., 1982, Vision, Freeman, San Francisco. Martin, M., 1994, "Perceptual Content", en Guttenplan 1994, pp. 463-471.

McDowell, J., 1994, Mind and World, Harvard University Press, Cambridge, Mass.

McLaughlin, B. y J. Cohen, en prensa, Contemporary Debates in Philosophy of Mind, Blackwell, Malden.

Peacocke, C., 1992, A Study of Concepts, MIT Press, Cambridge, Mass.

—. 1986, Thoughts: An Essay on Content, Basil Blackwell, Oxford. 
Perry, J., 1979, "The Problem of the Essential Indexical", Nô̂s, vol. 13, no. 1, pp. 3-21.

Richard, M., 1994, “What Isn't a Belief?", Philosophical Topics, vol. 22, nos. 1-2, pp. 291-318.

Searle, J., 1990, "Consciousness, Explanatory Inversion and Cognitive Science“, Behavioral and Brain Sciences, vol. 13, pp. 585-642. (Reimpreso en MacDonald y MacDonald 1995, pp. 331-355.)

Skidelsky, L., 2006, "Personal-Subpersonal: The Problems of Inter-Level Relations", Protosociology. Special Issue: Compositionality, Concepts and Representations II: New Problems in Cognitive Science, vol. 22, pp. 120-139.

- 2003, Representaciones mentales: ¿Eslabón entre el individuo y el mundo?, manuscrito de la tesis de doctorado, Facultad de Filosofía y Letras, Universidad de Buenos Aires, Argentina.

— de la competencia lingüística", Subjetividad y procesos cognitivos.

Skidelsky, L. y D. Pérez, 2005, "La distinción personal-subpersonal y la autonomía de la explicación de nivel personal en Dennett", Manuscrito, vol. 28, no. 1, pp. 77-112.

Speaks, J., 2005, "Is There a Problem about Nonconceptual Content?", Philosophical Review, vol. 114, no. 3, pp. 359-398.

Stich, S., 1978, "Beliefs and Subdoxastic States", Philosophy of Science, vol. 45, pp. 499-518.

Travis, C., 1994, "On Constraints of Generality", Proceedings of the Aristotelian Society, vol. 94, pp. 165-188.

- (comp.), 1986, Meaning and Interpretation, Blackwell, Oxford.

Weiskrantz, L., 1986, Blindsight: A Case Study and its Implications, Oxford University Press, Oxford.

Recibido el 4 de agosto de 2006; revisado el 26 de marzo de 2007; aceptado el 31 de marzo de 2007. 